\title{
Phosphorylation of BMK1 induces prostatic carcinoma cell proliferation by promoting entry into the $S$ phase of the cell cycle
}

\author{
YING XIONG $^{1 *}$, LUYING ZHANG $^{2 *}$ and TAO WANG ${ }^{1}$ \\ ${ }^{1}$ Department of Urology, First Affiliated Hospital of Yangtze University, Jingzhou, Hubei 434000; \\ ${ }^{2}$ Division of Anatomy, Hubei College of Chinese Medicine, Jingzhou, Hubei 434100, P.R. China
}

Received November 13, 2014; Accepted September 29, 2015

DOI: 10.3892/ol.2015.3909

\begin{abstract}
Big mitogen-activated protein kinase 1 (BMK1) is activated by mitogens and oncogenic signals, and is strongly implicated in tumorigenesis. In the present study, it was demonstrated that the activation of BMK1, but not extracellular signal-regulated kinase (ERK)1/2, can induce proliferation in prostate cancer cells. It was found that the proliferation of epidermal growth factor (EGF)-treated cells was accelerated by $40 \%$ compared with non-treated cells using a CCK 8 assay. In addition, cell cycle analysis using flow cytometry showed that the proportion of cells in the S phase increased significantly in the BMK1-activated PC-3 cells, suggesting that the activation of BMK1 promotes entry into the $\mathrm{S}$ phase of the cell cycle in prostate cancer cells. Furthermore, western blot analysis indicated that EGF-mediated activation of BMK1, but not ERK1/2, participates in the proliferation and cell cycle regulation in prostate cancer cells. Furthermore, the effects of cell cycle regulation by the activation of BMK1 were associated with the increased expression levels of cyclin A and cyclin E, whereas the expression of cyclin B and cyclin D1 was unchanged in this process. Therefore, the present study demonstrated that the activation of BMK1 can induce proliferation by promoting entry into the $\mathrm{S}$ phase through the upregulation of cyclin A and cyclin E expression levels in prostate cancer cells.
\end{abstract}

\section{Introduction}

Prostate cancer is the most common malignancy in the world, affecting 1 in 9 men $>65$ years of age. Currently, there is no effective cure for advanced-stage prostate cancer and it is the second leading cause of cancer-associated mortality in

Correspondence to: Dr Tao Wang, Department of Urology, First Affiliated Hospital of Yangtze University, 8 Hangkong Road, Jingzhou, Hubei 434000, P.R. China

E-mail:wangtao69@yangtzeu.edu.cn

${ }^{*}$ Contributed equally

Key words: BMK1, prostate cancer cells, cell cycle, cell proliferation, epidermal growth factor men $(1,2)$. Identification of novel endogenous factors responsible for the proliferation, migration and invasion of prostate cancer will facilitate our understanding of the progression of prostate cancer, and the development of novel approaches for its diagnosis and therapy.

There are four mitogen-activated protein (MAP) kinase pathways in mammalian cells: The extracellular signalregulated kinase $(E R K) 1 / 2$, Janus kinase, p38 and big mitogen-activated protein kinase 1 (BMK1) pathways (3-5). The BMK1 pathway was the last to be identified and the is least studied mammalian MAP kinase cascade. BMK1 is most similar to ERK1/2, as each contains the Thr-Glu-Tyr dual phosphorylation motif. However, BMK1 has a unique activating loop structure and an unusually large C-terminal non-kinase domain. The C-terminal half of BMK1 contains a nuclear localization signal that is critical for the nuclear localization of BMK1 (6). The ERK1/2 and BMK1 cascades are activated by mitogens and oncogenic signals, and are strongly indicated to be involved in tumorigenesis (3-5). Moreover, deregulated BMK1 signaling has been associated with properties of human malignancies, including the chemoresistance of breast tumor cells (7), the uncontrolled proliferation of erb-b2 receptor tyrosine kinase 2-overexpressing carcinomas (8), the metastatic potential of prostate tumor cells (9) and tumor-associated angiogenesis (10). Although the role of BMK1 has been demonstrated to be required for growth factor-induced cell proliferation and cell cycle regulation $(11,12)$, its biological significance for the development of prostate cancer remains elusive.

The present study sought to examine the biological functions of activated BMK1 in the cell proliferation and cell cycle regulation of prostate cancer cells.

\section{Materials and methods}

Cell culture. The prostate cancer PC-3 cell line and the normal prostate epithelial RWPE-2 cell line were obtained from the American Type Culture Collection (Manassas, VA, USA) and cultured in a $5 \% \mathrm{CO}_{2}$ humidified atmosphere at $37^{\circ} \mathrm{C}$. The cells were grown in Dulbecco's modified Eagle's medium (DMEM; Gibco; Thermo Fisher Scientific, Inc., Waltham, MA, USA) supplemented with $10 \%$ fetal bovine serum (Gibco; Thermo Fisher Scientific, Inc.) and 1:100 penicillin/streptomycin (Invitrogen; Thermo Fisher Scientific, Inc.). 
Antibodies and reagents. Rabbit monoclonal anti-ERK5 antibody (also known as BMK1-antibody; 1:3,000; ab40809), rabbit polyclonal cyclin D1 antibody (1:3,000; ab7958), mouse monoclonal cyclin E antibody (1:3,000; ab3927), mouse monoclonal cyclin A antibody $(1: 3,000 ;$ ab38) and mouse monoclonal cyclin B antibody $(1: 3,000 ;$ ab72) were obtained from Abcam (Cambridge, UK) and rabbit monoclonal anti-phospho-ERK1/2 antibody (1:4,000; \#4377) was purchased from Cell Signaling Technology, Inc. (Danvers, MA, USA). Rabbit polyclonal anti-EGF antibody (1:5,000; \#SAB2104809) was purchased from Sigma-Aldrich (St. Louis, MO, USA), while mouse monoclonal anti-GAPDH antibody (1:5,000: \#A01622-40) and mouse monoclonal actin antibody (1:5,000; \#A00702-100) were obtained from GenScript (Piscataway, NJ, USA). Horseradish peroxidase-labeled goat anti-mice immunoglobulin $\mathrm{G}$ (1:5,000; \#115-035-062) or goat anti-rabbit (1:3,000; \#111-035003) immunoglobulin $\mathrm{G}$ antibody was purchased from Jackson ImmunoResearch Laboratories, Inc. (West Grove, PA, USA). XMD8-92, a BMK1 inhibitor that specifically inhibits the phosphorylation of BMK1, but not the phosphorylation of ERK1/2, and PD184352, which is an ERK1/2 inhibitor that only blocks ERK1/2 but not BMK1 activated by EGF treatment, were purchased from Selleck Company (Houston, TX, USA). EGF was obtained from Sino Biological Inc. (Beijing, China). The final concentrations for the treatments of XMD8-92, PD184352, or EGF were $5 \mu \mathrm{M}, 1 \mu \mathrm{M}$ and $0.5 \mathrm{ng} / \mathrm{ml}$, respectively.

Establishment of a stable MEK5-expressing cell line. To establish the stable transfectant expressing the MEK5 protein, the RWPE-2 cells were transfected with pcDNA3.1(+)-MEK5 recombinant plasmid using Lipofectamine 2000 (Invitrogen; Thermo Fisher Scientific, Inc.) according to the manufacturer's protocols. After $48 \mathrm{~h}$, the transfected cells were selected for in DMEM supplemented with $400 \mu \mathrm{g} / \mathrm{ml}$ of G-418 antibiotic (Invitrogen; Thermo Fisher Scientific, Inc.) for 2 weeks, and then maintained in culture medium containing $200 \mu \mathrm{g} / \mathrm{ml}$ G-418. The RWPE-2 cells were transfected with pcDNA3.1(+) and selected for using G-418 treatment, as in the control cells.

Cell proliferation assay using cell counting kit (CCK)-8. The cells were seeded at $2 \times 10^{3}$ cells/well in phenol red-free DMEM (Gibco; Thermo Fisher Scientific, Inc.) with $10 \%$ fetal bovine serum (FBS; $100 \mu \mathrm{l} /$ well) in a 96-well culture plate. The growth rates of the cells were determined using the CCK- 8 assay (Beyotime Institute of Biotechnology, Haimen, China). A total of $10 \mu \mathrm{l}$ CCK-8 working solution was added to each well on days $1-5$, followed by incubation for $2 \mathrm{~h}$ at $37^{\circ} \mathrm{C}$, and the absorbance was finally measured at $450 \mathrm{~nm}$ using a model 3550 microplate reader (Bio-Rad Laboratories, Inc., Hercules, CA, USA).

Cell cycle analysis by flow cytometry. Nuclear DNA content was measured by propidium iodide (PI; Sigma-Aldrich) staining and fluorescence-activated cell sorting analysis according to the manufacturer's protocols. In brief, the cells were trypsinized and collected. Subsequent to three washes with phosphate-buffered saline (PBS), the cells were resuspended in $70 \%$ methanol and fixed in $4^{\circ} \mathrm{C}$ for $30 \mathrm{~min}$. The cells were then washed and resuspended in PBS containing $20 \mu \mathrm{g} / \mathrm{ml}$ RNase A and $50 \mu \mathrm{g} / \mathrm{ml} \mathrm{PI}$, and incubated on ice for $30 \mathrm{~min}$. Cell cycle analysis was performed in a Coulter Epics
XL flow cytometer using the CellQuest program (Beckman Coulter, Inc., Brea, CA, USA) with manually set regions for the $G_{0} / G_{1}, S$ and $G_{2} / M$ phases. Data from 10,000 cells were collected for each data file.

Western blot analysis. To prepare the protein extracts, the cells were washed with PBS and harvested in $1 \mathrm{ml}$ PBS. Following centrifugation, the cells were resuspended and extracted in lysis buffer (Thermo Fisher Scientific, Inc.) for $30 \mathrm{~min}$ on ice. The lysates were centrifuged at $15,000 \mathrm{x}$ g for $10 \mathrm{~min}$ at $4^{\circ} \mathrm{C}$. The supernatants of the lysates were mixed with a 6X SDS sample buffer and boiled for $10 \mathrm{~min}$. The samples were separated in a $10 \%$ SDS polyacrylamide gel and then transferred to a polyvinylidene difluoride membrane (Millipore, Billerica, MA, USA). The membrane was blocked with $5 \%$ (w/v) skimmed dry milk and then blotted with the corresponding antibody in PBST buffer (0.1\% Tween-20 in PBS) with gentle shaking at room temperature for $2 \mathrm{~h}$. Subsequent to being washed with PBST four times, the membranes were incubated with the indicated secondary antibody. The signals were detected using a SuperSignal West Pico Substrate kit (Thermo Fisher Scientific, Inc.). The signals were measured by fluorescence intensity with ImageJ software (National Institutes of Health, Bethesda, MD, USA).

Human EGF ELISA assay. A total of $1 \times 10^{5}$ cells were seeded in a 24-well plate, and maintained in 0.5 ml DMEM cell culture medium with $0.5 \%$ FBS in each well. The tissue culture mediums were collected after $48 \mathrm{~h}$. The EGF concentrations were measured with a Human EGF ELISA Assay kit according to the manufacturer's protocol (Signosis Inc., Santa Clara, CA, USA).

Reverse transcription-quantitative polymerase chain reaction $(R T-q P C R)$. Total RNA was extracted from the cells using TRIzol $^{\mathrm{TM}}$ reagent (Invitrogen; Thermo Fisher Scientific, Inc.). RT-qPCR was performed using a SYBR PrimeScript RT-PCR kit (Takara Bio Inc., Otsu, Japan) on a Rotor-Gene 6000 Real-Time Genetic Analyzer (Corbett Life Science; Qiagen, Inc., Valencia, CA, USA) according to the manufacturer's protocols. The primer sequences of cyclin A, cyclin B1, cyclin D1, cyclin E and GAPDH are shown in Table I. The PCR protocol included a denaturation program $\left(95^{\circ} \mathrm{C}\right.$ for $2 \mathrm{~min}$ ), followed by 40 cycles of an amplification and quantification program $\left(95^{\circ} \mathrm{C}\right.$ for $5 \mathrm{sec}$ and $55-57^{\circ} \mathrm{C}$ for $\left.30 \mathrm{sec}\right)$ and a melting curve program $\left(55-95^{\circ} \mathrm{C}\right.$, with a $0.5^{\circ} \mathrm{C}$ increment each cycle). Each sample was replicated three times.

Statistical analysis. All results were analysis by SPSS statistical software, version 10.0 (SPSS, Inc., Chicago, IL, USA) and presented as the arithmetic mean \pm standard error of the mean. Student's t-test was performed for statistical analysis. $\mathrm{P}<0.05$ was considered to indicate a statistically significant difference.

\section{Results}

Activation of the ERK/MEK5/BMK1 pathway induces prostate cell proliferation. To determine the biological functions of BMK1 in prostate cancer, the expression levels of BMK1 were determined in prostate cancer PC-3 cells and normal 
Table I. Primer sequences for reverse transcription-quantitative polymerase chain reaction assay.

\begin{tabular}{|c|c|c|}
\hline Name & Forward sequence & Reverse sequence \\
\hline Cyclin A & 5'-atgagaccggetttccegca-3' & 5'-cccetggccacaggtcctcc-3' \\
\hline Cyclin B1 & $5^{\prime}$ atggcgetccgagtcaccag-3' & 5'-ctctggcactggctcagaca-3' \\
\hline Cyclin D1 & $5^{\prime}$ atggaacaccagctcctgtg-3' & 5'-ctgcaggcggctctttttca-3' \\
\hline Cyclin E & 5'-atgccgagggagcgcaggga-3' & 5'-ggatggtgcaataatccgag-3' \\
\hline GAPDH & $5^{\prime}$-atggggaaggtgaaggtcgg-3' & 5'-gccagtggactccacgacgt-3' \\
\hline
\end{tabular}

A

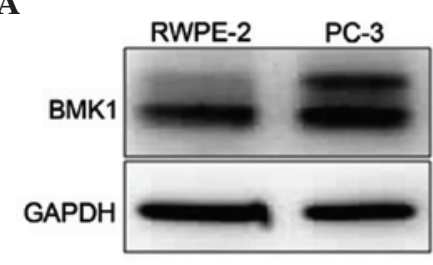

C

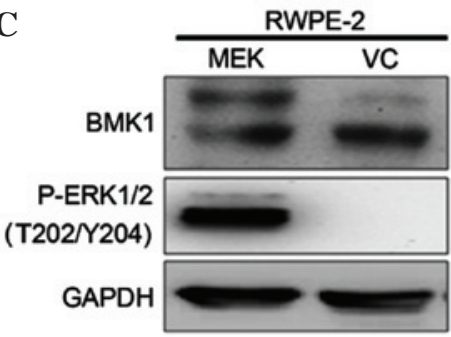

B

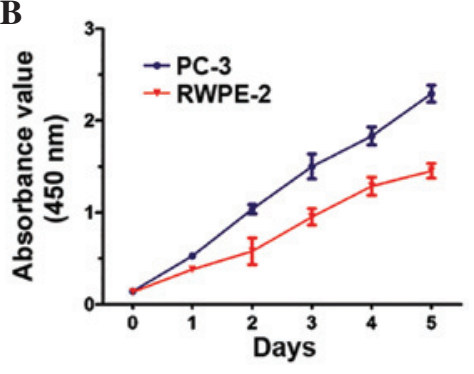

D

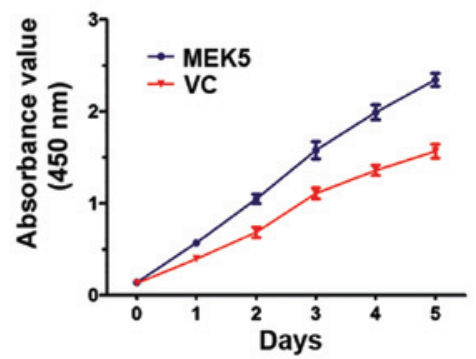

Figure 1. Activation of the ERK/MEK5/BMK1 pathway induces proliferation in prostate cells. (A) The determination of the expression levels of BMK1 in PC-3 and RWPE-2 cells by western blot analysis. (B) The proliferation of PC-3 and RWPE-2 cells. (C) The RWPE-2 cells were stably transfected with pcDNA3.1(+)-MEK5 and the pcDNA3.1(+) vector. The expression levels of BMK1 and phosporylated (P)-ERK1/2 were analyzed by western blotting. (D) The overexpression of MEK5 induced proliferation in the RWPE-2 cells. The proliferation of the MEK5 stably-expressed RWPE-2 and the control were measured by cell counting kit (CCK)- 8 assay at the indicated times (1,2,3,4 and 5 days). The mean \pm standard error of the mean for triplicates are shown for the CCK- 8 assay experiment. Western blot analysis using anti-GAPDH antibody was used to detect the GAPDH expression level, which served as an internal control. $\mathrm{VC}$, vector control.

A

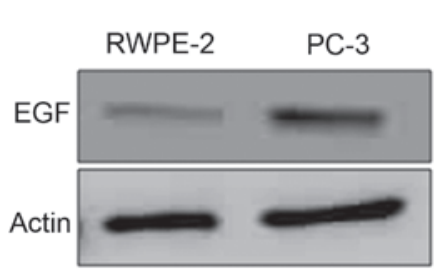

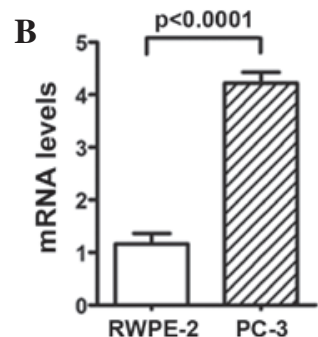

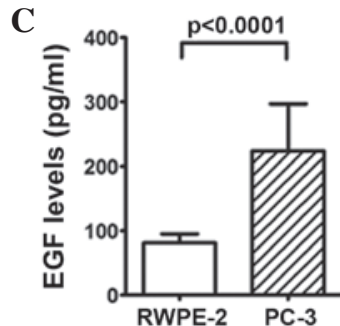

Figure 2. Epidermal growth factor (EGF) expression levels in RWPE-2 and PC-3 cells. The determination of the expression levels of EGF protein in the PC-3 and RWPE-2 cells by (A) western blot analysis, (B) reverse transcription polymerase chain reaction and (C) ELISA assay.

prostate epithelial RWPE-2 cells. It was found that these two cell lines each express BMK1 protein. However, the expression level of phosphorylated BMK1 in the PC-3 cells was much higher than that in the RWPE-2 control cells, suggesting that the expression of phosphorylated BMK1 may play a role in prostate cancer (Fig. 1A). Next, the proliferation of the PC-3 and RWPE-2 cells was measured. Notably, the proliferation rate of the PC-3 cells was significantly higher than that of the RWPE-2 cells (Fig. 1B). These results encouraged the further investigation of the biological functions of the activation of BMK1 in prostate cancer.

To investigate the association between phosphorylated BMK1 and proliferation, the expression level of phosphorylated BMK1 was upregulated in the RWPE-2 cells, and then 
A

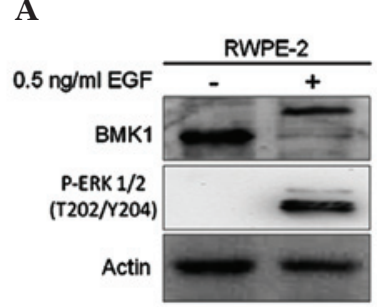

B

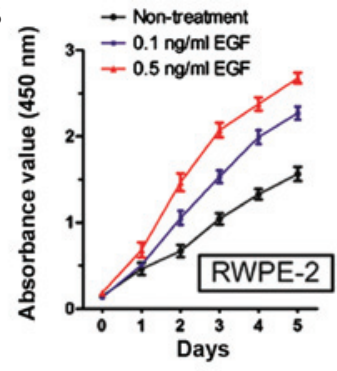

C

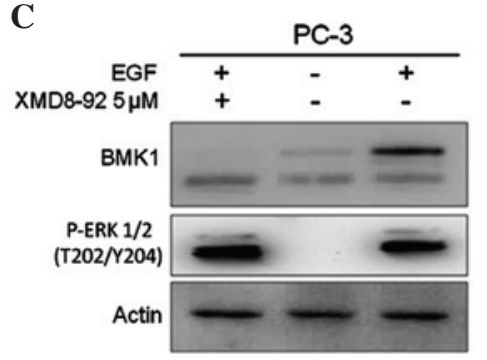

D $-0.5 \mathrm{mg}$ mI EGF

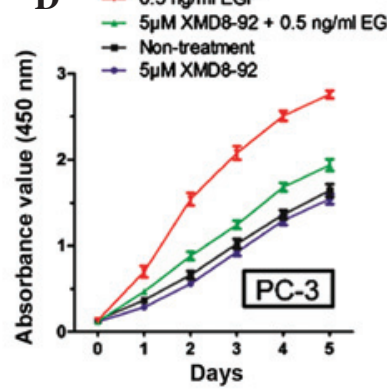

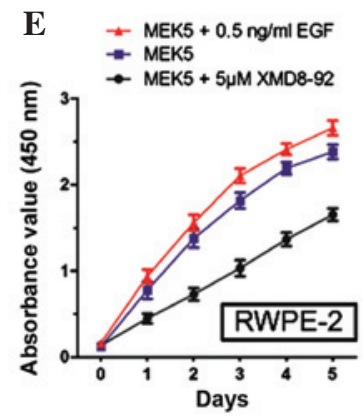

Figure 3. Epidermal growth factor (EGF)-mediated BMK1 activation induces the proliferation of RWPE-2 cells. (A) The phosphorylation of BMK1 or ERK1/2 in the RWPE-2 cells with or without the treatment of $0.5 \mathrm{ng} / \mathrm{ml}$ EGF. (B) The proliferation induced by $0.1 \mathrm{ng} / \mathrm{ml}$ or $0.5 \mathrm{ng} / \mathrm{ml}$ EGF treatment in PC-3 prostate cancer cells. (C) XMD8-92 significantly suppressed the EGF-stimulated phosphorylation of BMK1 and ERK1/2 in the PC-3 cells. All the samples were detected by western blot analysis. The proliferation induced by (D) $0.5 \mathrm{ng} / \mathrm{ml}$ EGF or (E) MEK 5 overexpression was inhibited by the treatment with $5 \mu \mathrm{M}$ XMD8-92 in the PC-3 and RWPE-2 cells, respectively. Data were obtained from three replicate experiments for the cell counting kit-8 assay.

A

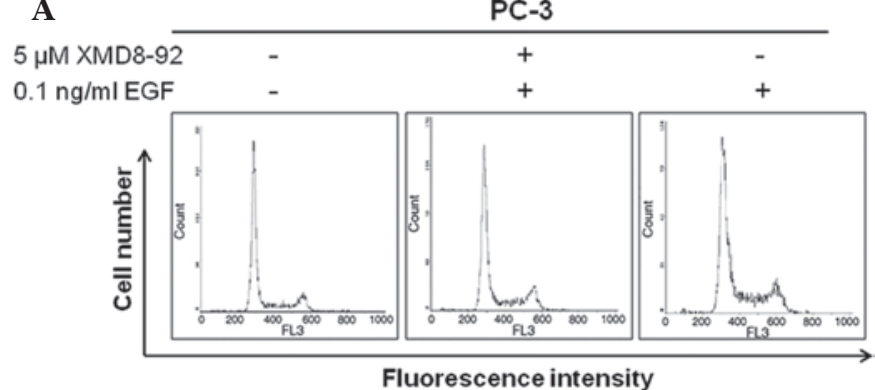

B

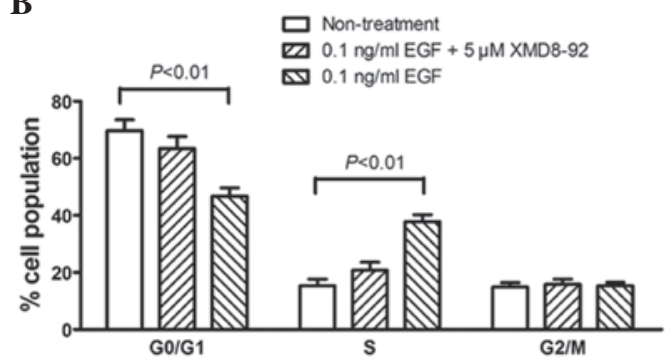

Figure 4. Activation of BMK1 promotes entry into the S phase of the cel cycle. (A) The PC-3 cells with different treatments were stained with propidium iodide and then analyzed by flow cytometry. (B) The histograms in panel A were analyzed to determine the percentage of cells in each phase of the cell cycle.

the proliferation of the cells with or without the activation of BMK1 was measured. It is known that the overexpression of MEK5 can activate the ERK/MEK/BMK1 pathway in vitro (13). Therefore, phosphorylated BMK1 or ERK1/2 was found in the stable MEK5-overexpressing RWPE-2 cells (Fig. 1C). Moreover, it was found that the proliferation increased by $>50 \%$ as measured by CCK- 8 assay in a culture time ranging from 1 to 5 days. This was accompanied by the activation of ERK/MEK5/BMK1, which was induced by the MEK5 overexpression in the RWPE-2 cells, suggesting that ERK/MEK5/BMK1 activation may promote cell proliferation (Fig. 1D).

EGF-mediated activation of BMK1 induces proliferation in prostate cancer cells. Since EGF has also been described as an activator of the ERK/MEK5/BMK1 pathway (11), the present study next tested whether EGF expression was involved in the cell proliferation of the prostate cancer cells. The analysis of EGF protein expression level in the PC-3 and RWPE-2 cells found a higher expression level in the PC-3 cells (Fig. 2A). RT-qPCR analysis and ELISA assays confirmed this result for mRNA and protein expression, respectively $(\mathrm{P}<0.0001$; Fig. 2B and C), suggesting that the higher expression level of EGF protein in the prostate cancer cells may be associated with cell proliferation. Furthermore, it was also found that the stimulation of $0.5 \mathrm{ng} / \mathrm{ml}$ EGF (Sino Biological, Inc., Beijing, China) could significantly activate the phosphorylation of BMK1 and ERK1/2 in the RWPE-2 cells (Fig. 3A), which is consistent with a previous study in HeLa cells (14). Next, the proliferation of the RWPE-2 cells with or without the treatment using different concentrations of EGF protein (0.1 and $0.5 \mathrm{ng} / \mathrm{ml}$ ), was measured. It was found that the proliferation of the RWPE-2 cells treated with EGF was much higher than that of the non-treated cells. Also, the proliferation was increased in a dose-dependent manner according to the EGF concentration (Fig. 3B).

Since either the ERK or the BMK1 pathway was activated by EGF treatment, further studies were performed to confirm which was involved in the proliferation of the prostate cancer cells. Following the treatment with EGF and/or $5 \mu \mathrm{M}$ XMD8-92, (Fig. 3C), it was found that the proliferation of the 
A

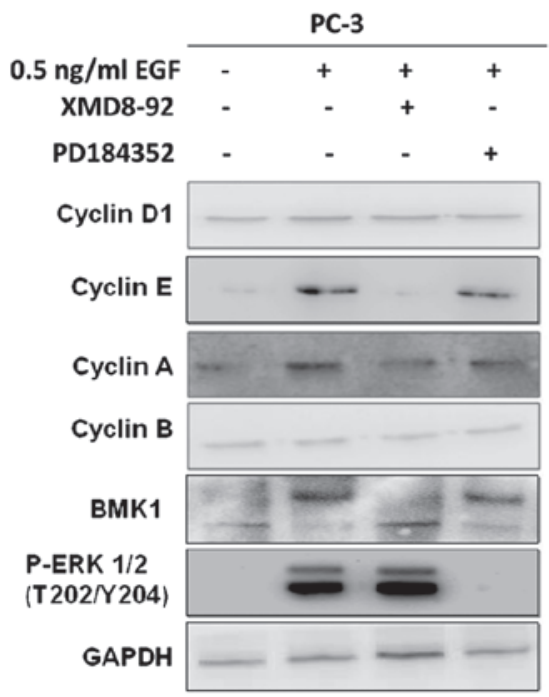

B

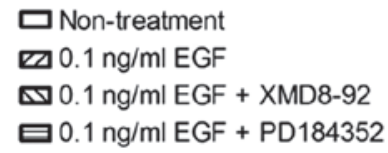

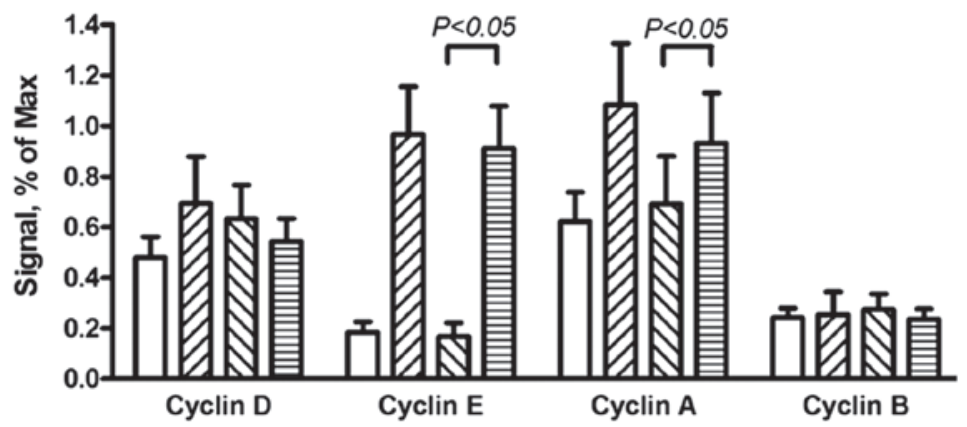

Figure 5. Epidermal growth factor (EGF)-mediated BMK1 activation promotes the entry into S phase, associated with upregulation of cyclin expression (A) The determination of the protein levels of cyclins associated with $\mathrm{G}_{1} / \mathrm{S}$ transition in the PC-3 cells without treatment, or with $0.5 \mathrm{ng} / \mathrm{ml}$ EGF, $0.5 \mathrm{ng} / \mathrm{ml}$ EGF plus $5 \mu \mathrm{M}$ XMD8-92, or $0.1 \mathrm{ng} / \mathrm{ml}$ EGF plus $1 \mu \mathrm{M}$ PD184352 treatment. The cells were lysed and equal amounts of protein from each sample were analyzed by western blotting. (B) Quantitative analysis of the intensity of the band corresponding to the expression level of cyclins.

PC-3 cells was increased by $0.5 \mathrm{ng} / \mathrm{ml}$ EGF and suppressed by $5 \mu \mathrm{M}$ XMD8-92. This suggested that EGF-mediated BMK1 activation induced the proliferation of the PC-3 cells (Fig. 3D). Similarly, the treatment with $5 \mu \mathrm{M}$ XMD8-92 significantly suppressed the proliferation in the MEK5 overexpressed RWPE-2 cells (Fig. 3E). All these results indicated that BMK1 activation induced by either MEK5 overexpression or EGF stimulation is essential for the proliferation of prostate cancer cells.

EGF-mediated BMK1 activation promotes entry into the $S$ phase in association with the upregulation of cyclin expression. To determine whether the proliferation of BMK1-activated cells was due to cell cycle regulation at certain phase(s), flow cytometry analysis was performed based on DNA content in nuclei stained with PI. The proportions of cells in the $G_{0} / G_{1}$, $\mathrm{S}$ and $\mathrm{G}_{2} / \mathrm{M}$ phases for the $0.5 \mathrm{ng} / \mathrm{ml}$ EGF-stimulated PC-3 cells were $46.8,37.8$ and $15.4 \%$, respectively, whereas those for the non-treated cells were $69.7,15.4$ and $14.9 \%$, respectively $(\mathrm{P}<0.01)$. Moreover, the percentages of cell populations in the EGF/XMD8-92 treated PC-3 cells were similar to those in non-treated cells (Fig. 4A and B). These results indicated that the proportion of cells in the $\mathrm{S}$ phase was significantly increased, accompanied by a decrease in the cell proportion in the $\mathrm{G}_{0} / \mathrm{G}_{1}$ phase, when compared with the non-treated cells. This suggested that the cell proliferation induced by activated BMK1 may be involved in the promotion of the entry into the S phase.

It is known that cell cycle progression is regulated using a complex network of positive or negative cell cycle regulatory molecules (14). Cyclin D and cyclin E are essential for the control of the cell cycle at the $\mathrm{G}_{1} / \mathrm{S}$ transition, while cyclin $\mathrm{A}$ is associated with the control of the cell cycle at the $G_{1} / S$ and $\mathrm{G}_{2} / \mathrm{M}$ transitions, and cyclin $B$ is involved with the $G_{2} / M$ transition only. Therefore, the expression levels of these cyclin proteins were examined by western blot analysis to investigate the molecular mechanisms involved in cell proliferation and cell cycle regulation induced by phosphorylated BMK1 in PC-3 cells. As expected, the expression levels of cyclin A and cyclin $\mathrm{E}$ (associated with the $\mathrm{G}_{1} / \mathrm{S}$ transition) were significantly increased, and the quantitative analysis indicated that expression levels were 2- and 10-fold higher, respectively, in the EGF-treated cells. This suggested that EGF-mediated BMK1 activation can promote the $G_{1} / S$ transition through upregulation of the expression levels of cyclins A and E. Furthermore, the EGF cells stimulated with XMD8-92 or PD184352, an ERK1/2 inhibitor, only blocked ERK1/2 but not BMK1 activation by EGF treatment, were also determined to reveal which pathway was involved in this process (Fig. 5A and B).

\section{Discussion}

Prostatic adenocarcinoma is the most frequently diagnosed type of malignancy in American men, accounting for $>35 \%$ of male cancers (15), with $20 \%$ of patients eventually succumbing to the disease. BMK1 promotes tumor development not only by inhibiting the tumor suppressor (16), but also by supporting tumor angiogenesis $(10,17,18)$, tumor metastasis $(19-21)$ and the chemoresistance of tumor cells (7). Furthermore, the knockout of BMK1 in various tissues of mice has been shown to have no marked effect on the development, behavior, reproduction and aging of the animals (10), suggesting that BMK1 should be an attractive target for pharmaceutical intervention in cancer therapy. The present study demonstrated that EGF-mediated BMK1 activation in prostate cancer is associated with cellular proliferation by promoting entry into the $S$ phase of the cell cycle. Furthermore, it was also found that the expression levels of cyclin A and cyclin $\mathrm{E}$ are regulated by the activation of BMK1 in this process.

In the present study, the level of phosphorylation of BMK1 in the PC-3 cells was higher than that in the RWPE-2 cells, suggesting that the higher proliferation of the PC-3 cells may 
be associated with the phosphorylated BMK1 (Fig. 1A and B). Following the activation of BMK1 induced by the overexpression of MEK5 in the RWPE-2 cells (Fig. 1C), it was found that this proliferation phenotype was also induced in MEK5-overexpressing cells. This can promote the activation of the ERK/ MEK5/BMK1 pathway (13), suggesting that the activation of BMK1 or ERK1/2 may play a role in the proliferation of the prostate cancer cells (Fig. 1D). Different expression levels of EGF protein were also noted in the RWPE-2 and PC-3 cells (Fig. 2). The EGF treatment was found to be an ERK/MEK5/ BMK1 pathway activator in the RWPE-2 cells (Fig. 3A). Furthermore, this proliferation can be inhibited by XMD8-92, suggesting that the activation of BMK1, not ERK1/2, induces the proliferation in the prostate cancer cells (Fig. 3D and E). Taken together, these results suggest that the high expression of EGF may result in this proliferation through the induction of activated BMK1 in the prostate cancer cells.

Since cell proliferation is usually associated with cell cycle regulation, a flow cytometry analysis was performed to measure the proportion of cells in the various cycle phases. A higher proportion of $\mathrm{S}$-phase cells indicated that the activated BMK1 increased $\mathrm{G}_{1} / \mathrm{S}$ transition in the cell cycle progression, which lead to the cell proliferation in the prostate cancer cells (Fig. 4A and B). As is known, cyclins A, $B, D$ and $E$ are all involved in the $G_{1} / M$ transition (14). Therefore, western blot analysis revealed that the expression levels of cyclins A and E were increased significantly. By contrast, there was no apparent change in the levels of cyclins B and D (Fig. 5). Moreover, the total RNA of EGF-stimulated cells and non-treated cells was extracted, and further RT-qPCR analysis demonstrated that the levels of cyclins $A$ and E mRNA were also markedly increased (data not shown). In previous studies, BMK1/MEK5 has been found to induce AP-1-mediated transcription (9), and BMK1 can suppress the functions of promyelocytic leukemia protein, which is associated with the transcription factors through phosphorylation $(16,22)$. This suggests that the effects of BMK1 activation on prostate cell proliferation and cell cycle progression may be a transcriptional event.

In summary, the present data provided evidence to indicate the functions of BMK1 in the cell proliferation and cell cycle regulation of human prostate cancer cells. The association between EGF and BMK1 activation in proliferation and cell cycle regulation were also clarified, suggesting the significance of a high expression level of EGF in human prostate cancer. However, further investigation in required into the novel mechanisms underlying proliferation and cell cycle regulation, further factors that may be involved in the EGF/BMK1 pathway, and the effects of BMK1 on tumor angiogenesis, differentiation and tumor metastasis in prostate cancer cells. Ongoing studies on BMK1 function may lead to the identification of an effective approach for treating human prostate cancer.

\section{Acknowledgements}

This study was supported by the startup fund from the First Affiliated Hospital of Yangtze University (Jingzhou, China).

\section{References}

1. Debes JD and Tindall DJ: Mechanisms of androgen-refractory prostate cancer. N Engl J Med 351: 1488-1490, 2004.

2. Schröder FH: Progress in understanding androgen-independent prostate cancer (AIPC): A review of potential endocrine-mediated mechanisms. Eur Urol 53: 1129-1137, 2008.

3. Chang L and Karin M: Mammalian MAP kinase signalling cascades. Nature 410: 37-40, 2001

4. Raman M, Chen W and Cobb MH: Differential regulation and properties of MAPKs. Oncogene 26: 3100-3112, 2007.

5. Johnson GL and Lapadat R: Mitogen-activated protein kinase pathways mediated by ERK, JNK and p38 protein kinases. Science 298: 1911-1912, 2002.

6. Lee JD, Ulevitch RJ and Han J: Primary structure of BMK1: A new mammalian map kinase. Biochem Biophys Res Commun 213: 715-724, 1995.

7. Weldon CB, Scandurro AB, Rolfe KW, Clayton JL, Elliott S, Butler NN, Melnik LI, Alam J, McLachlan JA and Jaffe BM: Identification of mitogen-activated protein kinase kinase as a chemoresistant pathway in MCF-7 cells by using gene expression microarray. Surgery 132: 293-301, 2002.

8. Esparis-Ogando A, Díaz-Rodriguez E, Montero JC, Yuste L, Crespo $\mathrm{P}$ and Pandiella A: Erk5 participates in neuregulin signal transduction and is constitutively active in breast cancer cells overexpressing ErbB2. Mol Cell Biol 22: 270-285, 2002.

9. Mehta PB, Jenkins BL, McCarthy L, Thilak L, Robson CN, Neal DE and Leung HY: MEK5 overexpression is associated with metastatic prostate cancer and stimulates proliferation, MMP-9 expression and invasion. Oncogene 22: 1381-1389, 2003.

10. Hayashi $M$ and Lee JD: Role of the BMK1/ERK5 signaling pathway: Lessons from knockout mice. J Mol Med (Berl) 82: 800-808, 2004.

11. Kato Y, Tapping RI, Huang S, Watson MH, Ulevitch RJ and Lee JD: Bmk1/Erk5 is required for cell proliferation induced by epidermal growth factor. Nature 395: 713-716, 1998.

12. Hayashi M, Tapping RI, Chao TH, Lo JF, King CC, Yang Y and Lee JD: BMK1 mediates growth factor-induced cell proliferation through direct cellular activation of serum and glucocorticoid-inducible kinase. J Biol Chem 276: 8631-8634, 2001.

13. Cameron SJ, Abe J, Malik S, Che W and Yang J: Differential role of MEK5alpha and MEK5beta in BMK1/ERK5 activation. J Biol Chem 279: 1506-1512, 2004.

14. John PC, Mews M and Moore R: Cyclin/Cdk complexes: Their involvement in cell cycle progression and mitotic division. Protoplasma 216: 119-142, 2001.

15. Greenlee RT, Hill-Harmon MB, Murray T and Thun M: Cancer statistics, 2001. CA Cancer J Clin 51: 15-36, 2001.

16. Yang Q, Deng X, Lu B, Cameron M, Fearns C, Patricelli MP, Yates JR III, Gray NS and Lee JD: Pharmacological inhibition of BMK1 suppresses tumor growth through promyelocytic leukemia protein. Cancer Cell 18: 258-267, 2010.

17. Hayashi M, Fearns C, Eliceiri B, Yang Y and Lee JD: Big mitogen-activated protein kinase 1/extracellular signal-regulated kinase 5 signaling pathway is essential for tumor-associated angiogenesis. Cancer Res 65: 7699-7706, 2005.

18. Pi X, Garin G, Xie L, Zheng Q, Wei H, Abe J, Yan C and Berk BC: BMK1/ERK5 is a novel regulator of angiogenesis by destabilizing hypoxia inducible factor lalpha. Circ Res 96: 1145-1151, 2005.

19. Sawhney RS, Liu W and Brattain MG: A novel role of ERK5 in integrin-mediated cell adhesion and motility in cancer cells via Fak signaling. J Cell Physiol 219: 152-161, 2009.

20. Sticht C, Freier K, Knöpfle K, Flechtenmacher C, Pungs S, Hofele C, Hahn M, Joos S and Lichter P: Activation of MAP kinase signaling through ERK5 but not ERK1 expression is associated with lymph node metastases in oral squamous cell carcinoma (OSCC). Neoplasia 10: 462-470, 2008.

21. Zhou C, Nitschke AM, Xiong W, Zhang Q, Tang Y, Bloch M, Elliott S, Zhu Y, Bazzone L and Yu D: Proteomic analysis of tumor necrosis factor-alpha resistant human breast cancer cells reveals a MEK5/Erk5-mediated epithelial-mesenchymal transition phenotype. Breast Cancer Res 10: R105, 2008.

22. Chatterjee A, Chatterjee U and Ghosh MK: Activation of protein kinase CK2 attenuates FOXO3a functioning in a PML-dependent manner: Implications in human prostate cancer. Cell Death Dis 4: e543, 2013. 